\title{
Transnational Social Movement Unionism als Vitalisierungsstrategie und Chance für Gewerkschafterinnen? Das Beispiel des Bekleidungssektors Bangladeschs
}

\section{Zusammenfassung}

Der Beitrag analysiert die Chancen und Grenzen eines transnationalen Social Movement Unionism (SMU) im Kontext des Bekleidungssektors Bangladeschs. SMU galt in Bangladesch insbesondere aufgrund des Prozesses der Depolitisierung zivilgesellschaftlicher Organisationen im Anschluss an die AidDependency des Landes als kaum realisierbar. Unberücksichtigt blieb hierbei aber weitestgehend die Rolle der Kategorie Geschlecht. Vor dem Hintergrund meines empirischen Materials, das zwischen 2010 und 2015 in Form von Interviews mit Angehörigen von Gewerkschaften und Labour-NGOs erhoben wurde, argumentiere ich, dass erste Ansätze von SMU erkennbar sind. Darüber hinaus zeigt der Blick auf den Bekleidungssektor Bangladeschs, dass das Potenzial dieses Organisierungskonzeptes sich insbesondere mit Blick auf die Arbeiterinnen und Gewerkschafterinnen offenbart. Ferner wird anhand dieses empirischen Beispiels die transnationale Rahmung des Arbeitsrechtsaktivismus verdeutlicht, der sich vom SMU der 1980er Jahre deutlich unterscheidet und als wegweisende Perspektive zu grenzüberschreitender Kooperation dienen kann. Nichtsdestotrotz hinterfragt der Beitrag die Notwendigkeit einer geographischen Lokalisierung des gegenwärtigen SMU und plädiert vielmehr für eine stärkere Berücksichtigung gesellschaftlicher Strukturkategorien innerhalb der Labour Studies sowie für eine größere Wachsamkeit gegenüber Organisierungsformen jenseits des traditionellen gewerkschaftlichen Musters.

Schlagwörter: Bangladesch, Bekleidungssektor, Social Movement Unionism, Gender

\section{Transnational Social Movement Unionism as a Revitalisation Strategy and Chance for Female Trade Unionists? A Case Study from Bangladesh's Ready-made Garment Sector}

\section{Abstract}

The paper analyses the opportunities and limits of Social Movement Unionism (SMU) in the context of the Bangladeshi garment sector. In the past, the concept of SMU was considered unrealistic in

\footnotetext{
* Elisabeth Fink, Gunta-Stölzl-Str. 23, 80807 München. E-Mail: lisfink@yahoo.com

** Artikel eingegangen: 30.8.2017, revidierte Fassung akzeptiert nach doppelt-blindem Begutachtungsverfahren: 8.3.2018
} 
Bangladesh, largely because of the de-politicization of civil society organizations, a result of the country's dependency on aid. The role of gender in relation to SMU in Bangladesh, however, remains on the whole ignored. Drawing on interviews with trade unionists and representatives of labour NGOs, conducted between 2010 and 2015, I argue evidence prevails to suggest that some aspects of SMU prevail. Furthermore, the role of female workers and trade unionists highlights the organizing potential of transnational SMU. In addition, the paper describes how the transnational framing of workers' rights activism, which differs from the SMU of the 1980s, might serve as an example for establishing solidarity across national borders. The contribution, however, questions the fixed geographical framings of current forms of SMU and instead emphasizes the importance of analyzing different social categories as well as paying more attention towards organizing beyond the traditional union model.

Key words: Bangladesh, Ready-made garment sector, Social Movement Unionism, Gender (JEL: F54, F66, J51, J83, L31, N35, P16)

\section{Einleitung: Social Movement Unionism in Bangladesch?}

Eine Kooperation zwischen transnationalen NGOs (TNGOs) ${ }^{1}$ und Gewerkschaften scheint im Kontext des Bekleidungssektors Bangladeschs zunächst nahezuliegen: Die schwachen Gewerkschaften können seit der Etablierung des Sektors in den 1980er Jahren keine nennenswerten Mitgliederzahlen vorweisen, (T)NGOs engagieren sich jedoch zunehmend im Sektor. Allianzen zwischen (T)NGOs und Gewerkschaften werden gleichwohl von Seiten der Gewerkschaften äußerst kritisch betrachtet. Sie befürchten eine NGOisierung der Gewerkschaftsbewegung. Im Zusammenhang mit der Aid-Dependency des Landes ${ }^{2}$ konnten sie beobachten, dass eine NGOisierung von Bewegungen mehrheitlich in deren Depolitisierung mündete (vgl. Nazneen, 2017; Kabeer, Mahmud \& Isaza Castro, 2010; Feldman 2003). Zusätzlich zu den ohnehin vorhandenen Spannungen zwischen diesen beiden Organisationsformen tritt im Kontext des Bekleidungssektors Bangladeschs demnach die ,double divide" (Anner \& Evans, 2004) zwischen dem globalen Norden und Süden folgendermaßen in Erscheinung: NGOs aus dem globalen Norden stehen unter dem Verdacht der hegemonialen Einhegung, des Boykotts und Protektionismus, des US-Imperialismus oder neokolonialer Dominanz. Gewerkschaften, die sich im linksradikalen politischen Spektrum verorten, lehnen eine Kooperation mit NGOs daher kategorisch ab. Nichtsdestotrotz liegen

1 Ich verwende die zunehmend geläufigere Bezeichnung, transnationale` statt, internationale “ NGO (TNGO statt INGO). Während der Begriff ,international ‘ im Forschungsfeld der Internationalen Beziehungen insbesondere für die Beziehungen zwischen Staaten verwendet wird, beschreibt der Begriff ,transnational ${ }^{\star}$ vielmehr Interaktionen auf nicht-staatlicher Ebene (vgl. Pries, 2010, S. 13). Die Bezeichnung TNGO wird somit im Gegensatz zu INGO dem zivilgesellschaftlichen und somit nicht-gouvernementalen Charakter dieser Organisationsform gerechter. Gleichwohl handelt es sich bei diesen Abgrenzungen (zivilgesellschaftlich vs. gouvernemental) um Idealtypen. Beispielsweise stehen NGOs vielfach in einem direkten finanziellen Abhängigkeitsverhältnis zum Staat.

2 Zum Zeitpunkt seiner Gründung im Jahr 1971 war der Staat Bangladesch vollends von sogenannten Entwicklungsgeldern aus Ländern des globalen Nordens abhängig. Diese Abhängigkeit führte zu einer massiven Beeinflussung, wenn nicht gar Dominanz, nationaler (Entwicklungs-)Politiken durch die Geberländer und -institutionen (vgl. Sobhan, 1982; Van Schendel, 2009, S. 144ff.). 
aber auch hier positive Erfahrungen mit Transnational Advocacy-Netzwerken vor. Zudem eröffnen grenzüberschreitende gewerkschaftliche Kooperationen die Perspektive für übergreifende zivilgesellschaftliche Bündnisse, die nicht zwangsläufig mit einer Kompromittierung der gewerkschaftlichen Position einhergehen müssen. In diesem Zusammenhang ist ebenso hervorzuheben, dass in der Gewerkschaftslandschaft des Bekleidungssektors Bangladeschs gegenwärtig ein frauenpolitischer Durchbruch zu verzeichnen ist: Erstmals sind mehrere Führungspositionen von Frauen besetzt. Dieser Prozess geht in erster Linie auf das jahrzehntelange frauenbewegte Engagement der Arbeiterinnen und Gewerkschafterinnen zurück, wurde aber zudem von ausländischen Trade Union Support Organisations und (T)NGOs unterstützt. Es liegt folglich eine widersprüchliche und unübersichtliche Ausgangslage bezüglich transnationaler Kooperationen vor, die im Rahmen dieses Beitrags anhand der Frage nach den Chancen und Grenzen von Transnational Social Movement Unionism (TSMU) im Kontext des Bekleidungssektors Bangladeschs erörtert wird.

Vor dem oben skizzierten Hintergrund widerspreche ich der These von Zia Rahman \& Peter Langford (2010), dass SMU auf den Kontext Bangladeschs, und somit auch auf den Bekleidungssektor des Landes, nicht übertragbar sei. Die beiden Autoren kritisieren, dass dem Konzept eurozentrische Annahmen zugrunde lägen. Obgleich diese Kritikperspektive grundsätzlich nachvollziehbar ist, ignorieren Rahman und Langford allerdings ihrerseits nahezu gänzlich die Beschäftigtenstruktur des Bekleidungssektors in Bangladesch: Über 80 Prozent sind Frauen. Angesichts dieses offensichtlich ungleichen Geschlechterverhältnisses und dem weltweit bekannten Problem der unzureichenden Geschlechterdemokratie in Gewerkschaften, dem unter anderem durch SMU begegnet werden soll, ist diese Auslassung nicht nur erstaunlich, sondern vielmehr nachlässig, da sie unwillkürlich zu entsprechenden Leerstellen in der Analyse führt.

Im Gegensatz zu Rahman und Langford argumentiere ich, dass erste Ansätze von TSMU erkennbar sind und das Potenzial dieses Mobilisierungskonzeptes sich am Beispiel des Bekleidungssektors Bangladeschs insbesondere mit Blick auf die Arbeiterinnen und Gewerkschafterinnen offenbart. Ferner wird anhand dieses empirischen Beispiels die transnationale Rahmung des Arbeitsrechtsaktivismus verdeutlicht, der sich vom SMU der 1980er Jahre deutlich unterscheidet und als wegweisende Perspektive zu grenzüberschreitender Kooperation dienen kann. Der Beitrag unterstreicht folglich die Chancen von SMU und verdeutlicht darüber hinaus die Notwendigkeit, sowohl die (selbst-)kritische Perspektive des Ansatzes auf bisherige Forschungsweisen der Labour Studies als auch auf konventionelle gewerkschaftliche Praxen ernst zu nehmen. Andernfalls wird sein (nach wie vor vorhandenes) innovatives Potenzial bezüglich der Wiederbelebung von Gewerkschaften und der Bündelung emanzipatorischer zivilgesellschaftlicher Ressourcen schlicht verspielt. Trotz der Potenziale des Ansatzes treten im Kontext von TSMU in Bangladesch auch Herausforderungen und Ambivalenzen zutage, auf die im Folgenden eingegangen wird.

Der Beitrag basiert auf Empirie, die ich im Rahmen der Arbeiten an meiner Dissertation erhoben habe (Fink, 2018). Während der explorativen Forschungsphase im Jahr 2010 und der Haupterhebungsphase im Jahr 2012, die jeweils in Bangladeschs Hauptstadt Dhaka stattfanden, habe ich insgesamt 47 ExpertInneninterviews mit WissenschaftlerInnen sowie VertreterInnen von Gewerkschaften und NGOs durchgeführt. Im Jahr 2015 fand eine Rückmeldungsphase in Dhaka statt, während der ich die InterviewpartnerInnen an den zent- 
ralen Ergebnissen meines Dissertationsprojekts teilhaben ließ und mich zudem über die Entwicklungen im Anschluss an das Desaster von Rana Plaza informierte (für eine ausführlichere Darstellung der methodischen Vorgehensweise s. Fink, 2018). Bei dem Einsturz des Rana Plazas in Savar, einem Vorort Dhakas, kamen am 24. April 2013 mindestens 1.134 BekleidungsarbeiterInnen ums Leben (das Schicksal von 166 vermissten Personen konnte bis heute nicht abschließend geklärt werden) und 2.535 wurden zum Teil schwer verletzt (vgl. Moazzem \& Islam, 2015). Seitdem steht der Begriff ,Rana Plaza“ nicht nur für die verheerendste Fabrikkatastrophe Bangladeschs, sondern als Synonym für die ausbeuterische Bekleidungsindustrie im globalen Süden.

Der Artikel ist folgendermaßen aufgebaut: Als ersten Schritt erläutere ich mein Verständnis von SMU und rekapituliere Rahman und Langfords Kritik an dem Konzept. Um das schwierige Verhältnis von Gewerkschaften und NGOs in Bangladesch und die hiermit verbundenen Grenzen von TSMU verstehen zu können, folgt ein kurzer Rückblick auf deren Geschichte. Sodann wird auf die Position von Frauen in den Bekleidungsgewerkschaften Bangladeschs eingegangen, um das Potenzial von TSMU anhand ihrer Rolle verdeutlichen zu können. Anhand dieses empirischen Beitrags wird schlussendlich aufgezeigt, inwiefern Forschungen zu SMU von dem analytischen Instrumentarium der Gender Studies, wie z.B. der Intersektionalität, profitieren könnten.

\section{Social Movement Unionism in der Diskussion}

Der Begriff Social Movement Unionism (SMU) ist in den späten 1980er Jahren insbesondere mit Blick auf Länder des globalen Südens entstanden, in denen Gewerkschaften Teil einer breiten gesellschaftlichen Bewegung gegen autoritäre Regierungen waren (vgl. Waterman, 1993). Vor dem Hintergrund von Südafrikas COSATU (Congress of South African Trade Unions) und Brasiliens CUT (Central Única dos Trabalhadores-Unified Workers' Central) entstand die Hoffnung, dass diese Formen der Organisierung den Weg in die gewerkschaftliche Zukunft weisen könnten (vgl. ebd., S. 268). Angesichts der Krise der Gewerkschaften war ein wesentlicher Bestandteil dieser Perspektive, deren Agenda um Anliegen jenseits der Fabriktore zu erweitern, um somit deren gesamtgesellschaftliche Relevanz zu stärken. Vor dem Hintergrund der Geschichte des Scheiterns sozialistischer Projekte sollte die Revitalisierung der ArbeiterInnenbewegung durch neue Konzeptualisierungen von Protest, Widerstand und gesellschaftlichen Visionen erreicht werden, die gemeinsam mit Menschen entworfen werden, die üblicherweise nicht zum klassischen Klientel von Gewerkschaften zählen. Dazu zählen insbesondere die Angehörigen der sogenannten neuen sozialen Bewegungen (z.B. die Queer- und Frauenbewegung, Menschenrechts- und Umweltbewegung), die sich von der , alten“ ArbeiterInnenbewegung abwandten, da sie ihre Interessen dort entweder überhaupt nicht vertreten oder lediglich nachrangig behandelt sahen. Kurzum: Angestrebt wurde eine Demokratisierung und Revitalisierung von ArbeiterInnenbewegung und Gewerkschaften, die insbesondere durch eine Öffnung hin zu den neuen sozialen Bewegungen vonstattengehen sollte (vgl. ebd., S. 266-268). Darüber hinaus ist die explizit internationale Ausrichtung von SMU darauf angelegt, auf die veränderten wirtschaftlichen und politischen Rahmenbedingungen unter den Bedingungen der globalen 
ökonomischen Restrukturierung im Rahmen der neoliberalen Globalisierung adäquat zu reagieren (vgl. Waterman, 1993, S. 245-248).

Die (Watermansche) Definition von SMU offenbart den sowohl empirischen als auch normativen Charakter des Konzeptes. Während COSATU und CUT Ende der 1980er und zu Beginn der 1990er als empirische Referenzen einer neuen Organisierungsform innerhalb eines nationalen Rahmens galten, entsprach der internationalistische Anspruch des Konzeptes mehr einer Vision. Obgleich COSATU und CUT als Beispiele für SMU nunmehr historisch sind und das Konzept als solches von Anbeginn umstritten war (vgl. von Holdt, 2002), ist es nicht ad acta gelegt, sondern dessen Existenz wird angesichts regelmäßig auftretender Koalitionsbildungen zwischen Gewerkschaften und anderen zivilgesellschaftlichen Akteuren, immer wieder aufs Neue geprüft (vgl. bspw. Nowak, 2017; Serdar, 2012; Shlyk, 2009). Gegenwärtig postuliert Jörg Nowak (2017, S. 981) beispielsweise, dass aktuelle Formen von SMU sich vom ,alten' SMU insofern unterscheiden, als dass sie nicht mehr auf der nationalen Ebene auftreten, sondern vielmehr auf der lokalen und regionalen Ebene. Transnationale SMU gäbe es hingegen, so Nowak, äußerst selten (vgl. ebd., S. 983). Vor diesem Hintergrund kritisiert er Ansätze wie Networks of Labour (NOLA) (vgl. Zajak, Egels-Zandén \& Piper, 2017; Zajak, 2017), die versuchen einen neuen Analyserahmen für gegenwärtige Erscheinungsformen transnationaler Kooperation zwischen mehreren verschiedenen Akteuren zu entwickeln, als „,voluntaristisch“ (ebd., S. 983). Jenseits einer Diskussion über die Berechtigung normativer Forschung, die sich hieran entfachen ließ, irritiert an diesem Argument, dass SMU als zentrales Konzept, auf das Nowak rekurriert, selbst einen stark normativen Charakter aufweist. Darüber hinaus kann die Organisierung rund um den Bekleidungssektor Bangladeschs durchaus als Beispiel für einen transnationalen SMU dienen. Transnationale feministische Diskurse schufen hier ab den späten 1980er Jahren den Nährboden für gegenwärtige Solidarisierungen über nationalstaatliche Grenzen hinweg. Vor diesem Hintergrund plädiere ich zum einen für eine stärkere Berücksichtigung von sozialen Strukturkategorien, wie beispielsweise der des Geschlechts, sowie von transnationalen feministischen Diskursen innerhalb der Labour Studies. Zum anderen hinterfrage ich die Notwendigkeit einer geographischen Lokalisierung des gegenwärtigen SMU. Ist solch eine Festschreibung überhaupt möglich und falls ja, welchen analytischen Mehrwert hätten wir hiervon? Gewinnbringender erscheint mir vielmehr eine kontextsensible Analyse seiner jeweiligen Erscheinungsformen. Zentral ist hierbei jedoch gewiss - wie von Waterman intendiert -, eine große Wachsamkeit gegenüber Organisierungsformen jenseits des traditionellen gewerkschaftlichen Musters. Dieses bedeutet zweifellos, dass das zuweilen 'konservative' analytische Repertoire der Labour Studies einer Diversifizierung bedarf, was unter anderem auch anhand der Begriffsgeschichte des SMU-Konzeptes ersichtlich ist.

SMU ist seit seiner Entstehung in den späten 1980er Jahren Gegenstand vielfältiger Aneignungen und Umdeutungen geworden, was letztlich dazu führte, dass SMU zwar in aller Munde ist, jedoch selten dasselbe damit gemeint ist (vgl. Scipes, 2014). Der kleinste gemeinsame Nenner aller gegenwärtigen Interpretationen von SMU ist wohl, dass das Konzept den Versuch darstellt, auf die Krise der Gewerkschaften mit einer Öffnung hin zu den neuen sozialen Bewegungen zu reagieren (vgl. Waterman, 1993). Jenseits dieser Minimaldefinition ist jedoch beispielsweise umstritten, welche Rollen den unterschiedlichen Akteuren innerhalb von SMU zukommen. Scipes (2014) unterscheidet diesbezüglich zwischen 
der seiner Ansicht nach verkürzten nordamerikanischen Rezeption im Anschluss an Kim Moody sowie der Ausrichtung, die sich in der Tradition von Peter Watermans Konzeptualisierung verortet. Während erstere eine avantgardistische Rolle des Proletariats vorsieht, versteht letztere die neuen sozialen Bewegungen und die ArbeiterInnenbewegung als gleichberechtigt. Die Adaption von Moody (1997) von SMU beraubt den Ansatz folglich seines kritischen Impetus, in dem abermals eine Hierarchie zwischen den verschiedenen Bewegungen hergestellt wird. Demgegenüber knüpft mein Verständnis von SMU an Waterman an, jedoch verwende ich dennoch im Folgenden die Bezeichnung Transnational Social Movement Unionism (TSMU) statt den durch Waterman zuletzt verwendeten Begriff des NISU (New International Social Unionism) (vgl. Waterman, 2004). Zum einen, da die Bezeichnung, transnational' meiner Perspektive nach sowohl die Distanz zu früheren Internationalismen orthodox marxistischer Prägung deutlich macht, als auch die Wortpaarung von Transnational Social Movement den zivilgesellschaftlichen und bewegungsorientierten Fokus adäquater reflektiert. Zudem soll durch diese begriffliche Distanzierung deutlich werden, dass mein Verständnis von TSMU im Gegensatz zu Watermans weniger utopischvisionären Charakter hat. Waterman, der im Jahr 2017 verstarb, verwehrte sich zuletzt generell gegenüber einer empirischen Verwendung von SMU und bezeichnete bloße Allianzen zwischen Gewerkschaften und sozialen Bewegungen nicht als SMU, sondern imaginierte vielmehr deren Synthese (vgl. Waterman, 2004). Hiervon abweichend erkenne ich fruchtbare Allianzen zwischen diesen beiden Organisations- bzw. Bewegungsformen durchaus als SMU an. Kurzum: Ich verstehe TSMU als eine Mobilisierungsstrategie, die (transnationale) Allianzen zwischen Gewerkschaften und sozialen Bewegungen vorsieht. Die sozialen Bewegungen werden hierbei häufig in der institutionalisierten Form einer NGO repräsentiert. Das Ziel dieser Allianzen ist ein Prozess der Demokratisierung und (Re-) Vitalisierung von Gewerkschaften, der mit einer breiteren und nachhaltigeren Organisierung von Beschäftigten einhergeht.

In Watermans Definition von SMU ist ersichtlich, dass Forderungen der Frauenbewegung inkorporiert wurden. Fernab eines Business Unionism reichen die im Rahmen von SMU formulierten Ziele klar über den Arbeitsplatz hinaus. So gehört beispielsweise die gerechte Verteilung von Hausarbeit zwischen den Geschlechtern ebenso wie die Bekämpfung von Sexismus und Rassismus am Arbeitsplatz, in Gewerkschaften und hierüber hinaus, zu den Zielen von SMU (vgl. Waterman, 1993, S. 266). Nichtsdestotrotz zeichnet sich die übrige SMU- und Gewerkschaftserneuerungs-Literatur eher durch eine geringe Berücksichtigung der Kategorie Geschlecht aus. Ebenso nehmen Fragen der Gewerkschaftserneuerung in den Gender Studies eher eine randständige Position ein. In einem sehr lesenswerten Artikel geht Jan Kainer (2009) der Frage nach, warum zwischen der Gewerkschaftserneuerungs-Literatur und den Gender Studies bisher nur wenige Berührungspunkte existieren. Insbesondere die nichtvorhandene Resonanz aus den Gender Studies führt sie darauf zurück, dass die Idee von SMU für Frauen in Gewerkschaften alles andere als neu ist, sondern vielmehr von ihnen seit jeher praktiziert wird. Schließlich sind es, so Kainers Argument, insbesondere die frauenbewegten Gewerkschafterinnen, die versuch(t)en, Gewerkschaften für die Anliegen von Frauen, die bspw. auch über die Lohnarbeit hinausreichen, zu sensibilisieren und selbige unter anderem hierüber zu demokratisieren. Teil dieses Demokratisierungsprozesses war, analog zu gleichzeitig stattfindenden entsprechenden Entwicklungen in 
den Frauenbewegungen, den singulären Fokus auf (weiße Mittelschichts-)Frauen auszuweiten und die gesellschaftliche Wirkungsmacht von sozialen Kategorien wie ,Rasse', Migration, Behinderung und sexueller Orientierung ernst zu nehmen (vgl. Kainer, 2009, S. 26). Ein hierfür bevorzugtes Mittel war spätestens seit den 1970er Jahren, auf nationaler wie transnationaler Ebene, die Bildung von Koalitionen mit Bewegungsorganisationen und NGOs. Jenes Engagement sei bisher allerdings vom überwiegend männlichen Mainstream der Gewerkschaftserneuerungs-Literatur nicht entsprechend gewürdigt worden und folglich erscheine die Koalitionsbildung mit anderen zivilgesellschaftlichen Akteuren hier als ,innovative und neue' Idee (2009, S. 32). Ebenso macht Alicia Schmidt Camacho (1999) in einem Rückblick auf die Gewerkschaftsgeschichte der USA darauf aufmerksam, dass es in erster Linie die Angehörigen der Bürgerrechtsbewegung waren, die den begrenzten Wirkungsbereich des Business Unionism entlarvten. Folglich stellte SMU für gewerkschaftlich engagierte People of Colour (PoC) in den USA häufig die einzige Möglichkeit dar, um ihren Anliegen über das Vehikel der Bürgerrechtsbewegung Gehör zu verschaffen und in die Gewerkschaften hineintragen zu können (Schmidt Camacho, 1999, S. 94). Die Kritik lautet folglich, dass die Begründer von SMU die Etablierung eines vermeintlich neuen Ansatzes für sich reklamierten, den Feministinnen und $\mathrm{PoC}$ längst praktizierten. Hiermit ging einher, dass das Potenzial einer beispielsweise geschlechtersensiblen SMU-Perspektive für Mobilisierung und Organisierung lange schlicht nicht gesehen wurde, was wiederum zu der Krise der Gewerkschaften beitrug. Diese problematische Ausgangssituation findet sich auch in Bangladesch. Wie eingangs erwähnt, wurde die Frage der Übertragbarkeit von SMU auf den Kontext Bangladesch, bisher nur von Rahman und Langford diskutiert, die hierauf eine abschlägige Antwort geben. Sie kritisieren, dass SMU ein eurozentrisches Modell sei, das den durch Kolonialismus und Imperialismus geprägten Kulturen der industriellen Beziehungen in Ländern des globalen Südens nicht gerecht werde. Im Einzelnen lauten ihre zentralen zwei Kritikpunkte, dass erstens weder Gewerkschaften noch NGOs in Bangladesch ihre Existenz ohne die Unterstützung Dritter absichern können und ihre Autonomie, die als Voraussetzung einer Allianzbildung auf Augenhöhe im Rahmen von SMU gilt, daher nicht gegeben sei (vgl. Rahman \& Langford, 2010, S. 55). Die beiden Autoren postulieren zweitens, dass SMU einen Mittelschichts-Bias aufweise, und somit der durch Imperialismus, Kolonialismus und Neoliberalismus herbeigeführten brutalen Lebensrealität in Ländern des globalen Südens nichts Wirksames entgegenzusetzen habe. Initiativen der Gewerkschaftserneuerung dürften sich daher, so die Autoren weiter, einzig auf die Bekämpfung von transnationalen Unternehmen, den kapitalistischen Kernländern, und ihren supranationalen Verbündeten konzentrieren (vgl. ebd., S. 59).

Der erstgenannte Kritikpunkt von Rahman und Langford trifft den Kern der schwierigen Voraussetzungen für SMU in Bangladesch und wird im Folgenden eingehender diskutiert. Der zweite Punkt wiederum ist eine etwas verklausuliert formulierte Wiederauflage des Haupt- und Nebenwiderspruchsargument, das die Anliegen der neuen sozialen Bewegungen, so auch der Frauenbewegung, durch den Verweis auf deren vermeintliche Nachrangigkeit zu diskreditieren versucht. An dieser Stelle tritt das große Beharrungsvermögen innerhalb der Labour Studies zutage, unter dessen Vorzeichen SMU weniger einer tatsächlichen Öffnung hin zu sozialen Bewegungen, als vielmehr deren Subsumtion entspricht. Im Rahmen dieses Artikels wird hingegen aufgezeigt, wie erst durch die gezielte Adressierung 
des vermeintlichen Nebenwiderspruchs des ungleichen Geschlechterverhältnisses, Organisierungspotenziale aufgezeigt werden können, die einen breiten und nachhaltigen Widerstand gegen ausbeuterische Arbeitsverhältnisse ermöglichen. Über die Rehabilitierung der Idee von SMU für den Kontext Bangladeschs hinaus, besteht das Anliegen dieses Artikels folglich darin, den zentralen Stellenwert der Berücksichtigung der spezifischen Verortung der Beschäftigten für Mobilisierungs- und Organisierungspraxen zu unterstreichen. Mit Blick auf die Bekleidungsindustrie Bangladeschs bedeutet dies, nicht nur den sozialen Platzanweisern der Klasse und geographischen Lokalität, sondern zumindest ebenso der Kategorie Geschlecht einen angemessenen Platz in der Analyse einzuräumen. Letztlich kann so zudem verdeutlicht werden, inwiefern die Labour Studies von der Perspektive der Gender Studies profitieren könnten, in der die Bedeutung unterschiedlicher Strukturkategorien im Hinblick auf die Analyse von Macht- und Herrschaftsverhältnissen spätestens seit der Debatte um das Paradigma der Intersektionalität prominent diskutiert wird. Das Charakteristikum dieses Forschungsparadigmas besteht im Gegensatz zu einer additiven Analyse mehrerer Kategorien vielmehr in der Untersuchung ihrer Wechselwirkungen. Angestrebt wird hierbei die Wechselwirkungen dieser Kategorien auf sowohl der Mikro-, Meso-, als auch Makroebene zu untersuchen (vgl. Crenshaw, 1991; Lutz, Herrera Vivar \& Supik, 2010). Wird diese Perspektive auf den Bekleidungssektor Bangladeschs in seiner transnationalen Verwobenheit angelegt, kann m.E. folgende These im Hinblick auf Strategien kollektiver Aktion formuliert werden: Während die Verortung der Beschäftigten einerseits Mobilisierung aufgrund des hohen Grades der Ausbeutung, des desolaten Zustandes der Gewerkschaften sowie ihrer männlichen Dominanz erschwert, eröffnen andererseits (trans-) nationale zivilgesellschaftliche Diskurse Gelegenheitsfenster zur Organisierung (vgl. Fink, 2018).

\section{Zivilgesellschaft und NGOs in Bangladesch}

Neben der Unabhängigkeitsbewegung als zentrale (historische) zivilgesellschaftliche Größe des Landes weist Bangladesch eine lange karitative Tradition auf (vgl. Lewis, 2011). Naturkatastrophen und Hungersnöte sowie der Unabhängigkeitskrieg mit seinen vielen Opfern und Geschädigten trugen in Anknüpfung an diese Tradition letztlich zur Etablierung des modernen NGO-Sektors bei. Im Rahmen der Aid-Dependency und entwicklungspolitischen Lösungen wie Good Governance hatten an der Errichtung dieses Sektors internationale Entwicklungsorganisationen und ausländische NGOs einen großen Anteil. Somit entstand eine neue Generation von ,Entwicklungs-NGOs' und der NGO-Sektor erfuhr ab den 1990er Jahren einen regelrechten Boom (vgl. Karim, 2001). Obgleich anfangs viele NGOs an Zielen der sozialen Mobilisierung orientiert waren, gaben die meisten jedoch diesen Anspruch - bedingt durch rechtliche Einschränkungen, Vorgaben (transnationaler) Geldgeber oder die Furcht vor der Verärgerung mächtiger Akteure - im Laufe der Zeit auf und konzentrierten sich vollends auf die Vergabe von Mikrokrediten (vgl. Karim, 2016; Kabeer \& Haq Kabir, 2009; Feldman, 2003; Stiles, 2002). Darüber hinaus wird konstatiert, dass NGOs durch den Prozess der Institutionalisierung und Transnationalisierung zu einer allgemeinen Depolitisierung der Zivilgesellschaft beigetragen haben. Die Denkschule des „Developmen- 
talism“ (van Schendel, 1995, zitiert nach White, 1999, S. 325) habe so weit um sich gegriffen, dass beispielsweise Armut nur mehr als technisches Problem verstanden werde, dem es durch entsprechende ,Projekte' oder den Zugang zu Mikrokrediten zu begegnen gelte. Fragen der gesellschaftlichen Machtverhältnisse würden somit ausgeklammert. Den Beschäftigten der NGOs, die zumeist der oberen Mittelklasse angehören und internationale Ausbildungen genossen haben, wird zudem vorgeworfen, mit ihrer Arbeit in erster Linie den eigenen sozialen Statuserhalt zu verfolgen. Im Kontrast zu ihrem international überwiegend positiven Image werden NGOs in Bangladesch folglich in erster Linie als eigennützige Organisationen gesehen, die das Los der armen Bevölkerung als ,Business-Idee ' für das eigene Fortkommen instrumentalisieren (vgl. Lewis, 2011, S. 125). Zusätzlich zu diesem ohnehin miserablen Ruf von NGOs, kommt innerhalb der Gewerkschaftsszene überdies erschwerend hinzu, dass das US-amerikanische Solidarity Center ${ }^{3}$ im Bekleidungssektor lange Zeit die einzig aktive NGO darstellte. Das imperialistische Erbe des Solidarity Centers und sein teils konkurrierendes sowie überhebliches Auftreten gegenüber den Gewerkschaften Bangladeschs, nährten vielmehr bereits existierende bangladeschische Narrative des Imperialismus und der Verschwörung, anstatt selbige zu entkräften (vgl. Rahman \& Langford, 2014). Andere im Bekleidungssektor aktive (T)NGOs werden von den Gewerkschaften als Konkurrenz wahrgenommen. Die (T)NGOs würden sich zwar in karitativer Absicht den ArbeiterInnen zuwenden, etablierten aber hierdurch, so die zentrale Kritik, eine Servicementalität, durch die sie die Beschäftigten von den Gewerkschaften entfremden (vgl. Fink, 2014). Vor diesem Hintergrund meidet ein großer Teil der Gewerkschaften NGOs und betrachtet deren Engagement im Bekleidungssektor äußerst kritisch. Die Gewerkschaften Bangladeschs können wiederum ihrerseits mit keinem guten Image aufwarten.

\section{Gewerkschaften in der Bekleidungsindustrie}

Bangladeschs Gewerkschaften haben sektorenübergreifend folgende zentrale Probleme: Eine starke Anbindung an politische Parteien; einen hohen Grad der Fragmentierung der Gewerkschaftslandschaft; eine sehr geringe Organisierungs- und Unterstützungsbasis; die Arbeiterferne der Gewerkschaftsfunktionäre; ein Strategie- und Kompetenzdefizit; Korruption sowie die geringe Repräsentation von Frauen (vgl. Faruque, 2009; Badruddin, 2004; Dan-

3 Das Solidarity Center (SC), dessen offizielle Bezeichnung American Center for International Labor Solidarity (ACILS) lautet, ist eine ,Quasi-NGO‘, die durch den US-amerikanischen Gewerkschaftsdachverband AFL-CIO (American Federation of Labor and Congress of Industrial Organizations) ins Leben gerufen wurde. Das SC ist dafür bekannt, während des Kalten Krieges in den jeweiligen Länderbüros die außenpolitischen Interessen der US-Regierung vertreten zu haben und wurde dafür wiederholt des Gewerkschaftsimperialismus bezichtigt (vgl. Scipes, 2012). Seinen Etat bestreitet das SC zu 95 Prozent aus Regierungsmitteln, die über die entwicklungspolitische Ausführorganisation USAID (United States Agency for International Development) sowie die durch den Kongress finanzierte Stiftung NED (National Endowment for Democracy) an das SC ausgezahlt werden (vgl. Bass, 2012, S. 104). Die Bezeichnung NGO ist daher fraglich. In Bangladesch ist das SC seit Jahrzehnten sehr aktiv, gehörte zu den ersten (ausländischen) zivilgesellschaftlichen Akteuren im Bekleidungssektor und trug unter anderem wesentlich dazu bei, eine Gewerkschaft namens BIGUF (Bangladesh Independent Garment Workers Union Federation) zu gründen. BIGUF entstand auf Initiative von vier ehemaligen Arbeiterinnen und zeichnete sich von Anbeginn durch einen hohen frauenpolitischen Anspruch aus (vgl. bspw. Dannecker, 2002). 
necker, 2000). All diese Defizite finden sich auch in den Gewerkschaften des Bekleidungssektors wieder, die hierin kaum präsent sind. So wurde 2009 auf Basis einer Befragung geschätzt, dass in lediglich 120 von damals insgesamt 4.500 Fabriken eine Betriebsgewerkschaft existiert (vgl. Faruque, 2009, S. 24). Der Organisierungsgrad war somit mit weniger als 5 Prozent verschwindend gering. Zwar ist im Anschluss an Rana Plaza eine vergleichsweise hohe Zahl an Neuregistrierungen von Gewerkschaften zu verzeichnen, die bis zum Frühjahr 2017 auf 571 angestiegen ist (vgl. Hossain Ovi, 2017). Es wird jedoch vermutet, dass unter anderem aufgrund der nach wie vor existierenden starken Repression durch das Fabrikmanagement nur ein Bruchteil dieser Gewerkschaften tatsächlich aktiv ist (vgl. ebd.). Überdies wäre auch ein Verhältnis von insgesamt circa 570 aktiven Betriebsgewerkschaften zu gegenwärtig knapp 4.500 Bekleidungsfabriken (vgl. BGMEA, 2017) nach wie vor sehr gering. Dies bedeutet, dass selbst mehrere Jahre nach dem Einsturz von Rana Plaza, das auf dramatische Weise die Konsequenzen des Fehlens einer Arbeitnehmervertretung deutlich gemacht hat ${ }^{4}$, keine bedeutenden Erfolge in Bezug auf die Realisierung der Vereinigungsfreiheit zu verzeichnen sind. De facto sind Gewerkschaften innerhalb der Produktionsstätten also nach wie vor kaum präsent. Hieran hat bisher auch der Bangladesh Accord on Fire and Building Safety nichts Wesentliches ändern können, der unter dem Eindruck von Rana Plaza zwar in erster Linie auf Gebäudesicherheit abzielt, aber von globalen Gewerkschaften entworfen wurde und durchaus das Ziel der Involvierung und Stärkung von Gewerkschaften formuliert (vgl. Zajak, 2017; Anner, 2015).

Die von mir interviewten GewerkschafterInnen begrüßen die Unterstützung seitens transnationaler Advocacy-Netzwerke, wie beispielsweise der Clean Clothes Campaign deutlich. Ebenso sehen Sie einen großen Bedarf hinsichtlich von Capacity Building-Maßnahmen durch beispielsweise (T)NGOs. Jegliches weiteres Engagement dieser Akteure im Sektor betrachtet die Mehrheit vor dem Hintergrund der oben erläuterten Erfahrungen jedoch kritisch. Nichtsdestotrotz gehen manche Gewerkschaften unter anderem vor dem Hintergrund ihrer schwierigen finanziellen Lage Kooperationen mit (T)NGOs ein. Aufgrund des ohnehin geringen Organisierungsgrades und der unregelmäßigen Zahlung von Mitgliedsbeiträgen ist die finanzielle Situation der Gewerkschaften äußerst prekär. Ohne externe Unterstützungsgelder, wie beispielsweise Spenden, Zuwendungen von nahestehenden Parteien oder auch Projekte mit NGOs könnte die Arbeit kaum aufrechterhalten werden.

Zusammenfassend muss bezüglich der Situation von NGOs und Gewerkschaften in Bangladesch folglich festgehalten werden, dass die Bedingungen für die Realisierung von SMU schwierig sind: Erstens fallen NGOs, denen im normativen Modell von SMU die Rolle der politischen Impulsgeber zukommt, vielmehr mehrheitlich durch ihren apolitischen Charakter auf. Zweitens sind weder NGOs noch Gewerkschaften, wie von Rahman und Langford (2010) diagnostiziert, in dem Maße autonom, als sie beispielsweise Entscheidungen zu etwaigen Bündnisbildungen ohne die Rücksichtnahme auf ihre jeweiligen Unterstützer fällen könnten. Vor diesem Hintergrund ist drittens das Verhältnis zwischen NGOs und Gewerkschaften vielfach durch Argwohn und Konkurrenz gekennzeichnet. Nichtsdestotrotz

4 Am Tag vor dem Einsturz bemerkten die Beschäftigten große Risse im Gebäude und evakuierten es. Am folgenden Tag wurden sie von den Fabrikbesitzern unter Androhung des Entzugs ihres Monatslohns dazu genötigt, wieder ihre Arbeit im Gebäude aufzunehmen. In den Fabriken gab es keine Gewerkschaft, die auf das Recht auf einen sicheren Arbeitsplatz hätte pochen können. 
existieren aber vereinzelt, wie im folgenden Abschnitt aufgezeigt wird, Allianzen zwischen diesen Organisationsformen, die deutliche Schnittstellen mit dem Modell von SMU aufweisen.

\section{Frauen in den Bekleidungsgewerkschaften Bangladeschs}

Während Frauen in den 1990er Jahren in den Bekleidungsgewerkschaften kaum aktiv waren und keine Führungspositionen einnahmen (vgl. Dannecker, 2000, S. 34) entspricht dies gegenwärtig nicht mehr dem Status quo. Obgleich die Repräsentation von Frauen in Gewerkschaften nach wie vor gering ist (vgl. BILS, 2009, S. 8), wird seit einigen Jahren von einer neuen Generation innerhalb der Gewerkschaftsszene des Bekleidungssektors gesprochen. Diese Generation zeichnet sich dadurch aus, dass sie weiblich und parteipolitisch unabhängig ist sowie, im Gegensatz zu ihrer Vorgängergeneration der studierten männlichen Berufsgewerkschafter, vor ihrer gewerkschaftlichen Laufbahn selbst als Arbeiterinnen tätig war. Darüber hinaus steht diese Generation mehrheitlich mit ausländischen Labour Support-Organisationen und transnational agierenden Akteuren in Kontakt. Somit kann festgehalten werden, dass die Transnationalisierung und Feminisierung von Gewerkschaftsarbeit in einem deutlichen Zusammenhang steht. Gewerkschafterinnen, die sich in ihrer Rolle diskriminiert sehen, identifizieren in der transnationalen Unterstützung zum einen die Möglichkeit, frauenspezifische Angebote für Arbeiterinnen anzubieten und zum anderen ihrem marginalen Status innerhalb der Gewerkschaftsbewegung etwas entgegenzusetzen. Ausländische zivilgesellschaftliche Organisationen wiederum unterstützen insbesondere Gewerkschafterinnen, um sie in ihrem Kampf für mehr Geschlechterdemokratie innerhalb der Gewerkschaftsszene zu unterstützen. (Trans-)nationale zivilgesellschaftliche Diskurse der Frauenemanzipation und Geschlechterdemokratie eröffnen somit Gelegenheitsfenster zur Organisierung (vgl. Fink, 2018). Dass Kontakte mit transnationalen Akteuren jedoch keine zwingende Voraussetzung für eine gewerkschaftliche Laufbahn sind, ist ebenso zu konstatieren. Während der Erhebungsphase meiner Dissertation (2010-2015) stellten Frauen in fünf Bekleidungsföderationen das Amt der Vorsitzenden und ich hatte die Gelegenheit mit weiteren zehn Gewerkschafterinnen zu sprechen, die jeweils andere zentrale Führungsaufgaben, wie etwa die der stellvertretenden Vorsitzenden, in ihren Föderationen einnahmen. Fünf dieser Gewerkschafterinnen entwickelten ihre gewerkschaftliche Laufbahn in engem Kontakt mit transnationalen Organisationen. Die übrigen zehn wiesen zumindest anfänglich keinerlei derartige Verbindungen auf, was sich jedoch änderte, nachdem sie Positionen in Föderationen innehatten. Einige von ihnen nahmen an den Trainings- und Vernetzungskursen der bangladeschischen NGO INCIDIN (Integrated Community and Industrial Development Initiative) teil, die folglich in der Berufsbiographie dieser Gewerkschafterinnen jeweils besondere Erwähnung fand. INCIDIN versteht sich als bewegungsnahe Organisation und bietet seit 2002 ein Qualifizierungs- und Vernetzungsprogramm für weibliche Gewerkschaftsvorsitzende an. Dieses Programm wurde anfänglich durch die TNGO Oxfam im Rahmen der Kampagne Make Trade Fair unterstützt, die unter anderem auf die Verbesserung der Arbeitsrechtssituation von weiblichen Beschäftigten am südlichen Ende transnationaler Wertschöpfungsketten abzielt. Über das Angebot an die weiblichen Gewerkschafts- 
vorsitzenden hinaus, waren breit angelegte Capacity Building-Maßnahmen für insgesamt 22 Gewerkschaftsföderationen Bestandteil des Programms von INCIDIN, die mithilfe von Gender Mainstreaming-Komponenten, die Rolle von Frauen in Gewerkschaften nachhaltig stärken sollten. Gegenstand der Fortbildungen für die weiblichen Gewerkschaftsvorsitzenden waren Computerkurse sowie Schulungen über Öffentlichkeitsarbeit und das Arbeitsrecht. Das Ziel des Projektes beschränkte sich aber nicht auf die bloße Wissensvermittlung, sondern sollte ebenso einen Raum zur Vernetzung und zum Dialog bieten, um so zum einen der Fragmentierung der Gewerkschaftsszene entgegenzuwirken und zum anderen einen strategischen Austausch zwischen weiblichen Gewerkschaftsvorsitzenden zu ermöglichen. INCIDIN zielt zudem darauf $a b$, insbesondere Gewerkschafterinnen über die Grenzen von Bangladesch hinaus zu vernetzen und Koalitionen mit anderen Gewerkschaften und zivilgesellschaftlichen (Bewegungs-)Akteuren zu ermöglichen. Hierzu diente beispielsweise eine gemeinsame Reise von INCIDIN-MitarbeiterInnen und Gewerkschafterinnen zum vierten Weltsozialforum nach Mumbai im Jahr 2004. Ebenso konnte INCIDIN in der Vergangenheit Kontakte mit globalen und ausländischen Gewerkschaften, Stiftungen und JournalistInnen herstellen, die jeweils zu Einladungen der Gewerkschafterinnen in diverse Länder Europas und Asiens geführt haben.

Das Engagement von INCIDIN erfährt seitens der Gewerkschaften einhellige Zustimmung. Die von mir interviewten Gewerkschafterinnen betonen die Wichtigkeit des Dreiklangs von Qualifizierung, Vernetzung über Gewerkschaftsgrenzen hinweg und internationalem Austausch, von dem sie ihrer Aussage nach sehr profitieren. INCIDIN sticht folglich als positives Beispiel unter den übrigen im Bekleidungssektor aktiven (T)NGOs heraus. Obgleich die Kooperation von INCIDIN mit den Gewerkschaften eindeutig durch eine Unterstützungsleistung seitens der NGO gekennzeichnet ist und damit nicht dem Idealbild einer gleichberechtigten Partnerschaft im Sinne von SMU entspricht, sind doch andere zentrale Charakteristika des Konzepts erkennbar. Insbesondere sind hierbei der Versuch der geschlechterpolitischen Demokratisierung der Gewerkschaften sowie die Vernetzung über Gewerkschaftsgrenzen und nationale Grenzen hinaus zu nennen. Diesem Fokus in INCIDINs Arbeit geht die Einsicht voraus, dass insbesondere Gewerkschafterinnen zur Vitalisierung der Gewerkschaften beitragen können. Um im Einzelnen zu illustrieren, welche Impulse zur Gewerkschaftserneuerung von Frauen ausgehen, die im Rahmen von Koalitionen mit NGOs wie INCIDIN idealerweise positiv verstärkt werden könnten, wird im Folgenden ein Blick darauf geworfen, was die Gewerkschafterinnen dazu motiviert, sowie welche Organisierungsformen und Strategien sie anwenden, um sich innerhalb der männlich dominierten Gewerkschaften durchzusetzen.

\section{Organisierungsformen, Motivation und Strategien}

Die favorisierte Organisierungsform meiner Interviewpartnerinnen stellt die separate Organisierungsstrategie dar. Darüber hinaus organisieren sie sich vereinzelt auch außerhalb von Gewerkschaften. Unter ersterem ist eine Organisierung in separaten Strukturen innerhalb von bestehenden Gewerkschaften, wie beispielsweise der Etablierung von Frauenkomitees, zu verstehen (vgl. Broadbent \& Ford, 2008, S. 5), was zweifelsohne die gängigste Organisierungsstrategie von Frauen in Bangladeschs Bekleidungssektor darstellt (vgl. BILS, 2009, S. 13). Eine Organisierung außerhalb von Gewerkschaften ist etwa ein Engagement im 
Rahmen von (Frauen-)Arbeitsrechts-NGOs, die bestehenden Gewerkschaften insbesondere aufgrund ihrer männlichen Dominanz vorgezogen wird (vgl. ebd., S. 7). Diese Organisierungsform wird jedoch äußerst ambivalent betrachtet, was anhand des beruflichen Werdegangs von Uma Nilanjan ${ }^{5}$ anschaulich verdeutlicht werden kann. Nilanjan wandte sich zunächst frustriert von einer Gewerkschaft ab, um in einer Frauen-NGO zu arbeiten, die Arbeit dieser Organisation jedoch ebenso als defizitär erachtete und daher ihre eigene FrauenNGO gründete. Ihre Beweggründe schildert sie folgendermaßen:

"While working for the trade union I realized, that I can't help the girls and women properly because as a
trade unionist I am not supposed to interfere in family matters. The law doesn't allow this and my colleagues
in the trade union didn't support this either. But some of the girls have very problematic family matters,
which need to be solved. (...) I was also unhappy in the trade union because of our position as women in the
union and our rights, we felt so dwarfish there. We didn't get what we deserve [lacht]. Then I learned about
the work of NGOs, which work for women's empowerment and because I was so unhappy in the federation I
decided to work for a NGO. There I worked with distressed women and after having worked there for a
while, I noticed that the NGO actually gives up before they achieve the target, which they settled. For exam-
ple, if I want to achieve women's empowerment, I need to empower women to a certain level. But before the
NGO gained that, they gave up. They gave up because the project duration was limited to a certain period of
time. But women's empowerment isn't done in one year, you cannot simply stop after some time, it's a con-
tinuing process. That is actually the main difference between trade unions and NGOs. The trade union works
continuously, the NGO doesn't. But unfortunately, trade unions don't focus enough on women's empower-
ment."

Aus diesem Zitat geht unmissverständlich hervor, dass Nilanjan die Organisierung innerhalb einer Gewerkschaft im Grunde vorzieht, da ihr die fehlende Nachhaltigkeit sowie der marktförmige Projektcharakter in der Arbeit von NGOs insbesondere aus feministischer Perspektive widerstrebt. Gleichzeitig ist in ihrer Aussage ebenso die Ambivalenz gegenüber Gewerkschaften erkennbar, in deren Arbeit sie zwar die nötige Nachhaltigkeit sieht, jedoch programmatische Defizite identifiziert. Während die NGO sich der Lebens- und Arbeitssituationen von Frauen zwar zeitlich begrenzt aber dennoch in einer ganzheitlichen Perspektive widme, zeichne sich die Gewerkschaft durch eine mehrheitliche Ignoranz gegenüber den spezifischen Anliegen von weiblichen Beschäftigten aus. Diese Position Nilanjans steht beispielhaft für sowohl die Ambivalenz von Feministinnen gegenüber diesen beiden Organisationsformen als auch die kritische Reflexion des eigenen Engagements, die weltweit zu teils dilemmatischen Suchbewegungen nach der jeweils geeigneten Organisierungsform führt (vgl. bspw. Britwurm, Douglas \& Ledwith, 2012; Alvarez, 1999, 2009; Broadbent \& Ford, 2008). Obgleich sich vor diesem Hintergrund die Mehrheit der frauenbewegten bangladeschischen Gewerkschafterinnen dennoch für ein Engagement in einer Gewerkschaft entschied, zog Nilanjan es aufgrund der in der obigen Interviewpassage angesprochenen Missachtung von Frauen in Gewerkschaften vor, eine Frauen-NGO zu gründen. Der wesentliche Schlüsselmoment in ihrem Entscheidungsprozess war die Erinnerung an ihr Vorbild Mamata Banerjee, die im indischen Westbengalen Parteivorsitzende der Partei All India Trinamool Congress ist. Uma Nilanjan hofft, als Vorsitzende einer NGO diese Vorbildfunktion nun für jene Frauen, für die sie sich engagiert, einnehmen zu können. Dass weibliche Vorbilder in der Motivation und Arbeit der Gewerkschafterinnen eine große Rolle spielen, ist aus mehreren Interviews hervorgegangen. Zum Beispiel dienen die Vorbilder

5 Sämtliche Namen meiner Interviewpartnerinnen sind Pseudonyme. 
ebenso zur Untermauerung der eigenen politischen Haltung. Die Gewerkschafterin Tamina Robin reagiert beispielsweise auf den Versuch einiger ihrer Gewerkschaftskollegen, Arbeiterinnen über die Zuschreibung eines Sex-Arbeiterinnenstatus zu diskreditieren, mit folgender an Rosa Luxemburg angelehnten Argumentation:

"Some male trade union leaders, very few but some, said: 'Tamina-Apa ${ }^{6}$, lots of sex workers are working in the garment factories'. But our attitude is that sex workers are very exploited women of the society. Rosa Luxemburg was working a lot for them. She published a journal for sex workers. So our attitude is not that sex workers are bad or something like that. I think our attitude is completely different, our values are completely different."

Über den hohen Stellenwert von Vorbildern hinaus, erwähnen alle Gewerkschafterinnen den Zuspruch der Arbeiterinnen als zentrale Motivationsquelle für ihre Arbeit. Sämtliche Gewerkschafterinnen berichteten, dass sich Arbeiterinnen ihnen gegenüber - im Gegensatz zu ihren männlichen Kollegen - erstmals öffnen konnten und nur über sie den Zugang zu Gewerkschaften fanden. Dieser große Zuspruch bekräftigt die Gewerkschafterinnen darin, ihre Position, trotz der Diskriminierung innerhalb der Gewerkschaften, zu behaupten. Anika Begum war hierdurch sogar dazu motiviert, sich gegen den Widerstand ihres Ehemanns durchzusetzen, der ebenso in ihrer Gewerkschaft tätig ist:

"I was fighting and I never gave up [lacht]. In fact, I told my husband as his wife, as his relative, I always told him that he could leave anytime, that he could quit. Because the simple fact is that the workers want me."

Der große Zuspruch der Arbeiterinnen half Anika Begum letztlich auch dabei, sich einerseits gesellschaftlichen Restriktionen zu widersetzen, aber andererseits ebenso die sich widersprechenden gesellschaftlichen Rollenerwartungen, die an sie als Ehefrau und Gewerkschaftsfunktionärin herangetragen werden, zu versöhnen:

"There were several problems with my husband. We are husband and wife and in our country there are a lot
of prejudices. Initially, when I wanted to run for president, he was against it and he asked me: 'What will the
others say? What will they say if you are the president but I'm not? So I developed a logic for him and my-
self: Whenever I gave a speech at a rally I didn't do that as his wife. I told him that I will go there as a lady,
as a normal person, as a labour leader. And I will do all other related tasks on my own. I told him that I
wouldn't act as his wife. It was very good that the workers wanted me. This way, my husband was finally
forced to let me do my work."

In den obigen Zitaten klingt bereits an, dass die Gewerkschafterinnen den großen Zuspruch, den sie erfahren, auf die Tatsache zurückführen, dass sie Frauen sind und auf die Arbeiterinnen daher besser eingehen könnten. In der folgenden Interviewpassage wird nunmehr explizit benannt, dass die Gewerkschafterinnen sich insbesondere durch ihr Geschlecht und ihre hiermit zusammenhängende Sensibilität für die Anliegen der weiblichen Beschäftigten, zu ihrer Tätigkeit berufen fühlen:

"Actually, only very few of the federation leaders are female, most of them are male. But we, [Aufzählung der Namen von vier Gewerkschaftspräsidentinnen], we are all leaders. When we sit together and work together, they [männliche Gewerkschafter] do not like it. But women are changing, women are getting strong-

6 Der bengalische Begriff Apa bedeutet große Schwester und wird über die Bezeichnung verwandtschaftlicher Verhältnisse hinaus, in der Regel in freundschaftlichen oder beruflichen Kontexten für die respektvolle Anrede älterer Freundinnen oder Kolleginnen verwendet. 
er. They do not like that, but so what? We don't care! We are working together and we are working for the workers. 85 percent of the workers are female. We know their problems."

Aus der obigen Aussage, die während eines Vernetzungstreffens zwischen Gewerkschafterinnen im Büro der NGO INCIDIN getätigt wurde, treten zudem drei weitere Aspekte zutage: Erstens der Stellenwert des Zusammenhaltes zwischen den Frauen über die Gewerkschaftsgrenzen hinaus. Dies ist insbesondere vor dem Hintergrund der starken Fragmentierung der Gewerkschaften erwähnenswert und eröffnet eine Perspektive zu deren Überwindung durch die Frauensolidarisierung. Zweitens wird deutlich, dass es einer großen Kraftaufwendung, politischem Kampfeswille und der Vernetzung bedarf, um die eigene Position zu behaupten. Und drittens identifiziert Nilanjan einen gegenwärtigen Prozess der Stärkung unter Gewerkschafterinnen und womöglich auch Arbeiterinnen. Letztere Beobachtung Nilanjans ist insofern bezeichnend, als sie der ersten Generation der Bekleidungsgewerkschafterinnen und somit den ,Veteraninnen“ unter ihnen angehört. In den Aussagen ihrer Generation bezüglich der Anfänge ihrer gewerkschaftlichen Laufbahn dominiert ein Kampfvokabular, das verdeutlicht, dass ihr Weg äußerst steinig war. Daher betont Alia Rakib, die in den 1970er Jahre innerhalb einer Partei politisch sozialisiert wurde sowie zur selben Zeit bereits in der Frauenbewegung Bangladeschs engagiert war, und somit unter meinen Interviewpartnerinnen auf den längsten Zeitraum politischer Erfahrung zurückblicken kann:

"In the past we could not reach high positions in trade unions. As women we were deprived. When you see today's women, it is the result of our fight. We have reached this by doing a lot of struggle."

Demgemäß schildert sie, dass sie sich insbesondere zu Beginn ihres gewerkschaftlichen Engagements an manchen Fronten wortwörtlich bewaffnen musste:

"In the first federation in which I worked, when we went out on the streets for rallies we had to take sticks with us. We, the young women, we used to take wooden sticks with us so that we could defend ourselves. Because all the people on the streets were looking at us, they were teasing and harassing us. They used bad words against us. Men also tried to abuse us. (...) Not the men of our federation but the men on the streets."

Unter anderem aufgrund solcher Erfahrungen entschieden sich Rakib und die Kolleginnen ihrer Generation höchstwahrscheinlich auch für eine Strategie der ,Entweiblichung` oder vielmehr Dethematisierung ihres Geschlechts:

„No one thought that I am a woman [lacht]. From the beginning we, me and my female colleagues, did that. No one treated me as a woman. Everybody knows me as a political leader.”

Diese Strategie der Dethematisierung des Geschlechts, das zugunsten der Betonung der politischen oder gewerkschaftlichen Rolle in den Hintergrund gedrängt wird, offenbart einen deutlichen Strategiewandel zwischen der ersten und zweiten Generation von Gewerkschafterinnen im Bekleidungssektor. Während die erste Generation ihr Geschlecht als ,Störfaktor' ansah, den es möglichst zu verbergen galt, da er die politische Arbeit und das persönliche Fortkommen deutlich erschwerte, legitimiert die gegenwärtige Generation ihre Position mit Verweis auf ihr Geschlecht. Dieser Strategiewechsel ist in Verbindung mit nationalen und transnationalen Diskursen der Frauenbewegung zu verorten, die ,Frausein “ weniger als Defizit denn als wertvolle Ressource für das jeweilige Engagement umdeuteten (vgl. Nazneen, 2017; Ferree \& Tripp, 2006). Dieser Diskurswandel ist Bestandteil des bereits erwähnten Gelegenheitsfensters für Gewerkschafterinnen, das zum einen dazu dient, das En- 
gagement im Kontext Bangladeschs zu legitimieren und zum anderen eine vermehrte (transnationale) Koalitionsbildung und Unterstützung mit sich bringt.

Trotz ihrer Erfahrung des zuweilen auch mit physischen Mitteln ausgetragenen Kampfes gegen Männer, stellt Alia Rakib aber unmissverständlich klar, dass es nicht das Anliegen von Frauen sei, gegen einzelne Männer zu kämpfen, sondern vielmehr die gesellschaftliche ,Superstruktur' zu verändern, die im weiteren Verlauf des Gruppeninterviews von einer anderen Teilnehmerin ,Patriarchat' genannt wird:

\footnotetext{
"But now the women learned to fight, they learned about trade unions, they learned about society, they learned about family. What they learned is huge. Now we are always fighting. Now women know how to fight against the super structure. They don't want to go against men. They want to go against the super structure."
}

Rakib identifiziert folglich gegenwärtig sowohl eine umfassendere Bildung von Frauen, die sie dazu befähigt, gesellschaftliche Zusammenhänge zu erkennen und gegen sie anzugehen, als auch die Zunahme einer hiermit einhergehenden Selbstbehauptung. Dass die Selbstbehauptung innerhalb der Gewerkschaften nach wie vor vonnöten ist, betonen sämtliche Interviewpartnerinnen. Sie sehen sich mit einer Vielzahl an teils subtilen Mechanismen konfrontiert, die ihren Stand innerhalb der Organisationen erschweren. Hierzu gehört beispielsweise die Anberaumung von spätabendlichen Treffen, die Gewerkschafterinnen aufgrund familiärer Verpflichtungen, sicherheitsrelevanter Überlegungen oder/und gesellschaftlicher Restriktionen nicht wahrnehmen können. Ebenso werden Gewerkschafterinnen bei Versammlungen häufig just zu dem Zeitpunkt, an dem Diskussionen an Wichtigkeit gewinnen, gebeten, ihren Platz in der ersten Reihe für männliche Gewerkschafter frei zu machen. Zudem wird ein Phänomen der ,rhetorischen Modernisierung' (Wetterer, 2003) konstatiert, das darin besteht, verbal Zugeständnisse zu erbringen und sich in einer Gleichheitsrhetorik zu üben, dieses Gleichheitsversprechen dann aber nicht in die Tat umzusetzen (vgl. hierzu ebenso BILS, 2009, S. 15). Ebenso muss das geschlechterpolitische AgendaSetting nach wie vor erkämpft werden. In diesem Zusammenhang verweist Tamina Robin darauf, dass sie das Thema der sexuellen Belästigung am Arbeitsplatz zwar auf der Agenda ihrer Gewerkschaft platzieren konnte, dies aber nach wie vor der Überzeugungsarbeit bedarf:

"For example, the question of sexual harassment in the factory. Our organization raised this question for the first time and demanded that sexual harassment in the factory should be stopped. Whenever we raise this issue, the other leaders of different organizations they say: 'Oh Tamina-Apa do not raise this question, it is really bad. We should not talk about sexual harassment'. Then I ask: 'What do you mean? If I have a disease and if I want to cure that disease, I should mark the disease first and I should diagnose it and I should treat it. Otherwise how can I cure it?' It is a very big problem here, the sexual harassment."

Bezüglich der Position von Frauen in den Bekleidungsgewerkschaften Bangladeschs kann somit festgehalten werden, dass im Gegensatz zu den Anfängen der Industrie, gegenwärtig Frauen sowohl als Mitglieder innerhalb der Gewerkschaften als auch in Führungspositionen präsent sind. Der Zugang zu den Führungsämtern wurde, ebenso wie geschlechterpolitische Themensetzungen, hart erkämpft und beides bedarf nach wie vor der Verteidigung, obgleich innerhalb der Gewerkschaftsbewegung Zugeständnisse bezüglich der Repräsentation von Frauen erbracht wurden. Dies ist der stärkeren Präsenz und Gegenwehr von Gewerkschafterinnen zuzuschreiben, die insbesondere aufgrund von Bildungs- und Qualifizie- 
rungserfolgen sowie der Allianzbildung über Gewerkschaftsgrenzen hinweg gegenwärtig selbstbewusster auftreten. Kurzum: Es ist die Existenz einer neuen weiblichen, parteipolitisch unabhängigeren und transnational vernetzten Generation von Gewerkschafterinnen zu konstatieren. Überdies sind erste Ansätze eines (Transnational) Social Movement Unionism erkennbar, da beispielsweise die Kooperation mit progressiven NGOs, wie etwa INCIDIN gesucht wird, die ihrerseits in ihrer Arbeit von einer TNGO unterstützt wurde, um die Anliegen von Frauen in Gewerkschaften zu stärken.

\section{Resümee: Potenziale von TSMU im Bekleidungssektor Bangladeschs}

Entgegen Rahman und Langfords (2010) abschlägigem Urteil bezüglich der Realisierungsmöglichkeiten von (T)SMU in Bangladesch möchte ich abschließend vor dem Hintergrund der geschilderten Beobachtungen die Potenziale dieser Organisierungsform in folgenden fünf zentralen Punkte hervorheben: Erstens konnten Kooperationen zwischen Gewerkschaften und (T)NGOs, Frauen in ihrem ,Marsch durch die Gewerkschaften ' bereits unterstützen und somit zu deren Stärkung beitragen. Dies geht mit einer inhaltlichen Demokratisierung der Gewerkschaften einher, da die Gewerkschafterinnen beanspruchen, die Problemlagen der ArbeiterInnen in einer ganzheitlicheren Perspektive anzugehen, die auch Aspekte umfasst, welche zuvor aufgrund ihres vermeintlich privaten Charakters außen vor gelassen wurden. So ist die Skandalisierung von sexueller Gewalt am Arbeitsplatz oder die Thematisierung der Mehrfachbelastung von Frauen durch Fabrik-, Haushalts- und Fürsorgearbeit auf die Interventionen der Gewerkschafterinnen zurückzuführen.

Mit der stärkeren Präsenz von Frauen und der durch sie erwirkten thematischen Diversifizierung geht zweitens die Verhandlung und Transformation von Geschlechterbeziehungen und -verhältnissen in Gewerkschaften sowie hierüber hinaus einher. Hiervon zeugen sowohl Hinterfragungen der gesellschaftlichen Stigmatisierung von Sexarbeit seitens der Gewerkschafterinnen als auch die Auseinandersetzung um geschlechtlich kodierte Rollenzuschreibungen angesichts der Übernahme eines gewerkschaftlichen Postens. Die Nachhaltigkeit dieses Prozesses wird unter anderem durch die NGO INCIDIN im Rahmen von Gender Mainstreaming-Komponenten in Capacity Building-Maßnahmen versucht zu gewährleisten.

Drittens hat das Engagement von Trade Union Support Organisations und (T)NGOs zu wichtigen Qualifizierungserfolgen der Gewerkschafterinnen geführt. Durch vergleichbare Angebote könnte zukünftig die Rolle der ArbeiterInnen in den Gewerkschaften gestärkt und somit am ungleichen Kräfteverhältnis zu den studierten Berufsgewerkschaftern gerüttelt werden. Die Basisnähe der Gewerkschaften wäre hierdurch eher gegeben.

Hiermit zusammenhängend deutet viertens die gezielte Nachfrage seitens der Arbeiterinnen nach Gewerkschafterinnen auf eine größere Akzeptanz von Gewerkschaften und somit auf einen perspektivisch höheren Grad der Organisierung der weit überwiegend weiblichen Beschäftigten hin.

Fünftens birgt das Moment der Vernetzung und Frauensolidarisierung über gewerkschaftliche Grenzen hinaus, das durch die NGO INCIDIN Unterstuitzung findet, die Per- 
spektive der Überwindung der Fragmentierung der Gewerkschaftslandschaft, was letztlich wiederum mit einer Rehabilitierung und Stärkung der Gewerkschaften einhergehen könnte.

Trotz dieses Potenzials, das in Kooperationen von Gewerkschaften mit (T)NGOs liegt, bleiben abschließend ebenso die limitierenden Faktoren dieser Organisierungsstrategie festzuhalten: Zum einen existieren aufgrund des skizzierten Prozesses der Depolitisierung der bangladeschischen Zivilgesellschaft nur wenige Organisationen, deren Engagement mit dem von INCIDIN vergleichbar ist. Zum anderen müssen im Falle bestehender Kooperationen Gewerkschaften wie auch NGOs, angesichts vielfältiger Abhängigkeiten im transnationalen Gefüge (vgl. Zajak, 2017), Tendenzen der Depolitisierung oder gar Disziplinierung stetig entgegenarbeiten (vgl. Fink, 2018). TSMU vor diesem Hintergrund aber schlicht als Option für Bangladesch zu verwerfen, würde bedeuten, das oben skizzierte Potenzial dieser Mobilisierungsform für Organisierung und gewerkschaftliche Erneuerung nicht anzuerkennen und zudem Gelegenheitsfenster der Organisierung zu verspielen. Für die Labour Studies bedeutet dies zweierlei: Erstens muss der eindimensionale Fokus auf Beschäftigte als einer vermeintlich einheitlichen Interessensgruppe durch eine Analyse ihrer spezifischen Verortung mittels beispielsweise des Analyseparadigmas der Intersektionalität ersetzt werden. So konnten in diesem Beitrag durch die Differenzierung der Gewerkschaftsmitglieder nach Geschlecht, Alter, Klasse und geographischer Lokalität sowohl die Schwierigkeiten der Organisierung (männliche Dominanz der Gewerkschaften, desolater Zustand der Gewerkschaften bedingt durch Geschichte des Kolonialismus und Imperialismus), die Differenzen innerhalb der Gewerkschaften (Generations-, Geschlechter- und Bildungsunterschied) sowie die Chancen für Revitalisierung und Mobilisierung (Nachfrage nach Gewerkschafterinnen, thematische Diversifizierung durch Frauen, Solidarisierung über Gewerkschaftsgrenzen hinweg, transnationale Ressourcen und Gelegenheitsfenster) herausgearbeitet werden. Die gleichzeitige Berücksichtigung der Mikroebene (Verhandlung von geschlechtlich kodierten Identitäten) sowie der Meso- und Makroebene (Verfasstheit von Gewerkschaften und zivilgesellschaftlichen Organisationen, Geschlechterverhältnisse innerhalb dieser Organisationen, transnationale zivilgesellschaftliche Diskurse und labour governance) erlaubt hierbei, die unterschiedlichen Ebenen in Beziehungen zu setzen, sowie Wechselwirkungen, Diskursverschiebungen und nicht zuletzt Gelegenheitsfenster zur Organisierung, die sich aus einer Kongruenz nationaler und transnationaler Diskurse ergeben, zu identifizieren.

In Zusammenhang mit dieser analytischen Diversifizierung muss innerhalb der Labour Studies sowie der Gewerkschaftsbewegung zweitens eine tatsächliche Offenheit gegenüber sozialen Bewegungen praktiziert werden, die darin besteht, deren Anliegen als gleichberechtigt zu akzeptieren. Für Forschungen zu SMU bedeutet dies konkret, von der vermeintlich avantgardistischen Position von Gewerkschaften Abstand zu nehmen und sich gegenüber den demokratisierenden Interventionen, die von den Angehörigen der sozialen Bewegungen ausgehen, wachsam zu zeigen. In diesem Sinne sind Studien, die Organisierungsformen jenseits des traditionellen gewerkschaftlichen Musters zum Gegenstand haben, zu begrüßen (vgl. bspw. Nowak, 2017; Zajak, Egels-Zandén \& Piper, 2017). In dieser Annäherung an soziale Bewegungen, wäre es zudem im Sinne eines Akzeptanzgewinns seitens der zuvor von den Gewerkschaften und Labour Studies vielfach vernachlässigten Gruppen nicht zuletzt hilfreich, die historischen Vorläufer (der Watermanschen Prägung) von SMU, 
wie beispielsweise die Frauen- oder amerikanische Bürgerrechtsbewegung, als solche anzuerkennen und zu würdigen.

\section{Literatur}

Alvarez, S. E. (1999). The Latin American Feminist NGO 'Boom'. International Feminist Journal of Politics, 2, 181-209. doi: 10.1080/146167499359880

Alvarez, S. E. (2009). Beyond NGOization? Reflections from Latin America. Development, 52 (2), 175-184. doi: 10.1057/dev.2009.23

Anner, M. (2015). Stopping the Race to the Bottom. Challenges for Workers' Rights in Supply Chains in Asia. Berlin: Friedrich-Ebert-Stiftung.

Anner, M., Bair, J. \& Blasi, J. (2013): Towards Joint Liability in Global Supply Chains: Addressing the Root Causes of Labor Violations in International Subcontracting Networks. Comparative Labor Law and Policy Journal, 35 (1), 1-43.

Anner, M. \& Evans, P. (2004). Building Bridges Across a Double Divide: Alliances between US and Latin American Labour and NGOs. Development in Practice, 14 (1-2), 34-47. doi: 10.1080/0961452032000170613

Badruddin, U. (2004). The Emergence of Bangladesh: Class Struggles in East Pakistan, 1947-1958, Vol.1. Karachi: Oxford University Press.

Bass, G. N. (2012). Organized Labor and U.S. Foreign Policy: The Solidarity Center in Historical Context. FI Electronic Theses and Dissertations. Paper 752. Abgerufen von http://digitalcommons.fiu.edu/etd/752

BGMEA (2017). Trade Information. Abgerufen am 08.08.2017 von http://bgmea.com.bd/home/pages/TradeInformation.

BILS (Bangladesh Institute of Labour Studies). (2009). Women's Participation in Trade Unions in Bangladesh. Status, Barriers and Overcoming Strategies. Abgerufen von http://www.ilo.org/wcmsp5/groups/public/@asia/@ro-bangkok/@ilodhaka/_documents/publication/wcms_125374.pdf

Britwurm, A. O., Douglas, K. \& Ledwith, S. (2012). Women, Gender and Power in Trade Unions. In M. Williams \& S. Mosoetsa (Hrsg.), Labour in the Global South: Challenges and Alternatives for Workers (S. 41-64). Geneva: International Labour Office.

Broadbent, K. \& Ford, M. (2008). Introduction. In K. Broadbent \& M. Ford (Hrsg.), Women and Labour Organizing in Asia. Diversity, Autonomy and Activism (S. 1-15). Routledge: New York.

Crenshaw, K. (1991). Mapping the Margins. Intersectionality, Identity Politics, and Violence against Women of Color. Stanford Law Review, 43 (6), 1241-1299. doi: 10.2307/1229039

Dannecker, P. (2000). Collective Action, Organisation Building and Leadership. Women Workers in the Garment Sector in Bangladesh. Gender and Development, 8 (3), 31-39. doi: $10.1080 / 741923779$

Dannecker, P. (2002): Between Conformity and Resistance: Women Garment Workers in Bangladesh. Dhaka: Dhaka University Press.

Faruque, A. A. (2009). Current Status and Evaluation of Industrial Relation System in Bangladesh. ILO. Abgerufen von www.ilo.org/wcmsp5/groups/public/@asia/@robangkok/@sro-new_delhi/documents/ publication/wcms_123336.pdf

Fink, E. (2014). Trade Unions, NGOs and Transnationalisation: Experiences from the Ready-made Garment Sector in Bangladesh. ASIEN, 130, 42-59.

Fink, E. (2018). Transnationaler Aktivismus und Frauenarbeit. Social Movement Unionism in Bangladesch. Frankfurt/New York: Campus. 
Feldman, S. (2003). Paradoxes of Institutionalisation. The Depoliticisation of Bangladeshi NGOs. Development in Practice, 13 (1), 5-26. doi: 10.1080/0961452022000037955

Ferree, M. M. \& Tripp, A. M. (Hrsg.) (2006). Global Feminism. Transnational Women's Activism, Organizing, and Human Rights. New York: New York University Press.

Hossain Ovi, I. (23. April 2017). Garment Workers' Rights Still a Far Cry. Dhaka Tribune. Abgerufen von http://www.dhakatribune.com

Kabeer, N. \& Haq Kabir, A. (2009). Citizenship Narratives in the Absence of the Working Poor in Bangladesh, Working Paper 331, Institute of Development Studies at the University of Sussex Brighton, 1-65.

Kabeer, N., Mahmud, S. \& Isaza Castro, J. G. (2010). NGO's Strategies and the Challenge of Development and Democracy in Bangladesh, Working Paper 343, Institute of Development Studies at the University of Sussex Brighton, 1-66.

Kainer, J. (2009). Gendering Union Renewal: Women's Contributions to Labour Movement Revitalization. In J. R. Foley \& P. L. Baker (Hrsg.) (2009), Unions, Equity, and the Path to Renewal (S. 15-39). Vancouver: UBC Press.

Karim, L. (2001). Politics of the Poor? NGOs and Grassroots Political Mobilisation in Bangladesh, Polar, 24 (1), 92-107. doi: 10.1525/pol.2001.24.1.92

Karim, L. (2016). Resistance and its Pitfalls: Analyzing NGO and Civil Society Politics in Bangladesh. In D. Courpasson \& S. Vallas (Hrsg.), The SAGE Handbook of Resistance (S. 461-475). Thousand Oaks, CA: SAGE. doi: 10.4135/9781473957947.n25

Lewis, D. (2011). Bangladesh. Politics, Economy and Civil Society. Cambridge: Cambridge University Press. https://doi.org/10.1017/CBO9781139017138

Lutz, H., Herrera Vivar, M. T. \& Supik, L. (Hrsg.). (2010). Fokus Intersektionalität: Bewegungen und Verortungen eines vielschichtigen Konzeptes. Wiesbaden: VS Verlag für Sozialwissenschaften. doi: 10.1007/978-3-531-92555-4

Moazzem, K. \& Islam, A. (2015). Moving Beyond the Shadow of the Rana Plaza Tragedy. In Center for Policy Dialogue (Hrsg.), Search of a Closure and Restructuring Strategy. Fourth Monitoring Report. Abgerufen von http://cpd.org.bd/wp-content/uploads/2013/07/Fourth-Monitoring-Report-Rana-Plaza-TragedyFINAL-April-2015.pdf

Moody, K. (1997). Workers in a Lean World: Unions in the International Economy. London and New York: Verso.

Nazneen, S. (2017). The Women's Movement in Bangladesh. A Short History and Current Debates. Friedrich-Ebert-Stiftung, Dhaka.

Nowak, J. (2017). Mass Strikes in India and Brazil as the Terrain for a New Social Movement Unionism. Development and Change, 48 (5), 965-986. doi: 10.1111/dech.12320

Rahman, Z. \& Langford, T. (2010). The Limitations of Global Social Movement Unionism as an Emancipatory Labour Strategy in Majority World Countries. Socialist Studies, 6 (1), 45-64. doi: $10.18740 / \mathrm{S} 44597$

Rahman, Z. \& Langford, T. (2014). International Solidarity or Renewed Trade Union Imperialism? The AFL-CIO and Garment Workers in Bangladesh, Working USA: The Journal of Labor and Society, 17 (2), 169-186. doi: 10.1111/wusa.12106

Rock, M. (2001). The Rise of the Bangladesh Independent Garment-Workers' Union (BIGU). In J. Hutchison \& A. Brown (Hrsg.), Organising Labour in Globalising Asia (S. 27-47). London and New York: Routledge.

Schmidt Camacho, A. (1999). On the Borders of Solidarity: Race and Gender Contradictions in the "New Voice" Platform of the AFL-CIO. Social Justice, 26 (3 [77]), 79-102. 
Scipes, K. (2012). Globalization from Below. Labor Activists Challenging the AFL-CIO Foreign Policy Program. Critical Sociology, 38 (2), 303-323. doi: 10.1177/0896920511399938

Scipes, K. (2014). Social Movement Unionism or Social Justice Unionism? Disentangling Theoretical Confusion within the Global Labor Movement. Class, Race and Corporate Power, 3 (2), Article 9. Abgerufen von http://digitalcommons.fiu.edu/classracecorporatepower/vol2/iss3/9

Serdar, A. (2012). Strategies for Revitalizing Labor Movement: Union Organizing and Building Alliances with Community in Argentina. Economic and Industrial Democracy, 33 (3), 403-420. doi: 10.1177/0143831X11411326

Shlyk, A. (2009). Social Movement Unionism in Poland. Towards Revitalization of Organized Labor? Theory in Action, 2 (1), 158-184. doi: 10.3798/tia.1937-0237.08034

Sobhan, R. (1982). The Crisis of External Dependence. The Political Economy of Foreign Aid to Bangladesh. Dhaka: University Press Limited.

Stiles, K. (2002). International Support for NGOs in Bangladesh. Some Unintended Consequences. World Development, 30 (5), 835-846. doi: 10.1016/S0305-750X(02)00012-8

Van Schendel, W. (2009). A History of Bangladesh. Cambridge: Cambridge University Press. doi: 10.1017/CBO9780511997419

Von Holdt, K. (2002). Social Movement Unionism: The Case of South Africa. Work, Employment and Society, 16 (2), 283-304. doi: 10.1177/095001702400426848

Waterman, P. (1993). Social Movement Unionism: A New Model for a New World Order? Review, $16(3), 245-278$.

Waterman, P. (2004). Adventures of Emancipatory Labour Strategy As the New Global Movement Challenges International Unionism. Journal of World-System Research, 10 (1), 217-253. doi: 10.5195/JWSR.2004.315

Wetterer, A. (2003). Rhetorische Modernisierung. Das Verschwinden der Ungleichheit aus dem zeitgenössischen Differenzwissen. In G.-A. Knapp \& A. Wetterer (Hrsg.), Achsen der Differenz. Gesellschaftstheorie und feministische Kritik 2 (S. 286-319). Münster: Westfälisches Dampfboot.

White, S. C. (1999). NGOs, Civil Society, and the State in Bangladesh. The Politics of Representing the Poor. Development and Change, 30 (2), 307-326. doi: 10.1111/1467-7660.00119

Zajak, S. (2017): International Allies, Institutional Layering and Power in the Making of Labour in Bangladesh. Development and Change, 48 (5), 1007-1030. doi: 10.1111/dech.12327

Zajak, S., Egels-Zandén N. \& Piper, N. (2017). Networks of Labour Activism: Collective Action across Asia and Beyond. An Introduction to the Debate. Development and Change, 48 (5), 899 921. doi: 10.1111/dech.12336 


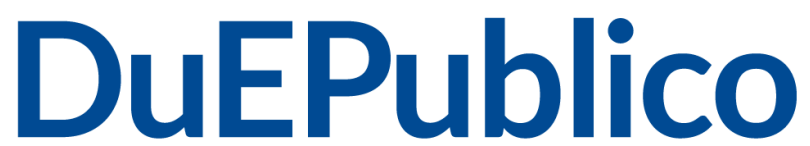

Duisburg-Essen Publications online offen im Denken

$\mathbf{U b} \mid \begin{aligned} & \text { universitäts } \\ & \text { bibliothek }\end{aligned}$

Dieser Text wird via DuEPublico, dem Dokumenten- und Publikationsserver der Universität Duisburg-Essen, zur Verfügung gestellt. Die hier veröffentlichte Version der E-Publikation kann von einer eventuell ebenfalls veröffentlichten Verlagsversion abweichen.

DOI: $\quad$ 10.3224/indbez.v25i2.04

URN: urn:nbn:de:hbz:464-20210401-113426-8 